\title{
Maternal and neonatal outcomes in Korean women with type 2 diabetes
}

\author{
Hye-Jung Jang ${ }^{1,}{ }^{,}$, Hee-Sook Kim ${ }^{2,}$, and Sung-Hoon Kim ${ }^{3}$
}

\begin{abstract}
${ }^{1}$ Department of Clinical Trials for Medical Devices, Severance Hospital, Yonsei University Health System, Seoul; ${ }^{2}$ Department of Nursing, Dongnam Health University, Suwon; ${ }^{3}$ Division of Endocrinology and Metabolism, Department of Medicine, Cheil General Hospital \& Women's Healthcare Center, Dankook University College of Medicine, Seoul, Korea
\end{abstract}

Received: March 28, 2016 Revised : August 4, 2016 Accepted: October 27, 2016

\section{Correspondence to} Sung-Hoon Kim, M.D. Division of Endocrinology and Metabolism, Department of Medicine, Cheil General Hospital \& Women's Healthcare Center, Dankook University College of Medicine, 17 Seoae-ro 1-gil, Jung-gu, Seoul 04619, Korea

Tel: +82-2-2000-7271

Fax: +82-2-2000-7147

E-mail: hoonie.kim@cgh.co.kr

*These authors contributed equally to this work.
Background/Aims: The purpose of this study was to compare maternal and neonatal outcomes in Korean women with type 2 diabetes and nondiabetic controls. Methods: We performed a retrospective survey of 200 pregnancies in women with type 2 diabetes $(\mathrm{n}=100)$ and nondiabetic controls $(\mathrm{n}=100)$ who delivered from 2003 to 2010 at Cheil General Hospital \& Women's Healthcare Center, Korea. We compared maternal characteristics as well as maternal and neonatal outcomes between groups matched by age, pre-pregnancy weight, body mass index, parity, and gestational age at delivery.

Results: The number of infants that were small for gestational age and the rate of major congenital malformations were not significantly different. However, women with type 2 diabetes showed a slightly higher risk for primary caesarean section ( $35.0 \%$ vs. $18.0 \%, p=0.006)$ as well as pre-eclampsia (10.0\% vs. $2.0 \%, p=0.017$ ), infections during pregnancy $(26.0 \%$ vs. $2.0 \%, p<0.001)$, neonatal weight $(3,370 \pm$ 552.0 vs. $3,196 \pm 543.3, p=0.025)$, large for gestational age (22.0\% vs. $9.0 \%, p=0.011)$, and macrosomia ( $15.0 \%$ vs. $5.0 \%, p=0.018)$ compared to nondiabetic controls.

Conclusions: Maternal and neonatal outcomes for women with type 2 diabetes were worse than those for nondiabetic controls. Diabetic women have a higher risk for primary caesarean section, pre-eclampsia, infections during pregnancy, large neonatal birth weight, large for gestational age, and macrosomia.

Keywords: Diabetes mellitus, type 2; Pregnancy outcome

\section{INTRODUCTION}

It has been predicted that by 2030 more than 360 million people will have diabetes mellitus [1]. Type 2 diabetes is the most common form of diabetes in women, and its prevalence in this group is continuing to rise $[2,3]$. Between 1999 and 2005 in the USA, the age- and race/ ethnicity-adjusted prevalence for pre-existing diabetes in pregnancies rose from $0.81 \%$ to $1.82 \%$ [4]. The interaction between ethnicity and pre-existing diabetes mel- litus has also been shown to affect pregnancy outcomes, and Asian populations tend to be affected more than other ethnicities [5].

Women with pregestational diabetes have been reported to have worse maternal and neonatal outcomes compared to nondiabetic controls [6]. Stamler et al. [7] showed that women with type 2 diabetes were more likely to have infections during pregnancy than women without diabetes. Indeed, the occurrence of almost all types of infections is increased in diabetic pregnancies 
[7]. Common infections include candida vulvovaginitis, urinary infections, respiratory tract infections, and puerperal pelvic infections. Stamler at al. [7] reported that the rate of infection was five times higher in a group of pregnant women with diabetes compared to nondiabetic pregnant women. Type 2 diabetes during pregnancy has been associated with a higher risk for adverse outcomes including stillbirth, perinatal mortality, congenital anomaly, macrosomia, shoulder dystocia at delivery, and the need for a caesarean section (C/S) [6,8-10]. Specifically, type 2 diabetes is associated with hyperglycemia and diabetes-related vascular complications, both of which affect the health of the mother and fetus.

Therefore, the aim of this study was to compare maternal and neonatal outcomes in Korean women with type 2 diabetes to nondiabetic controls.

\section{METHODS}

This was a case-control study to investigate maternal and neonatal outcomes of women with type 2 diabetes who were admitted to the delivery ward in Cheil General Hospital \& Women's Healthcare Center for the birth of their baby from 2003 to 2010 . The outcomes were compared to nondiabetic women who delivered during the same time period. Participants were matched by age, pre-pregnancy weight, body mass index (BMI), parity, and gestational age at delivery.

We performed a complete enumeration of 775 pregnancies in 150 women with pregestational type 2 diabetes mellitus and 625 nondiabetic controls using medical records from 2003 to 2010 at Cheil General Hospital \& Women's Healthcare Center, Korea. The criteria for inclusion in the study were Korean women with a singleton pregnancy. Nondiabetic women had a 1 hour plasma glucose $<130 \mathrm{mg} / \mathrm{dL}$ after a $50 \mathrm{~g}$ glucose challenge test. Subjects selected were 100 women with type 2 diabetes and 100 nondiabetic controls matched by age, pre-pregnancy weight, BMI, parity, and gestational age at delivery.

We compared the maternal characteristics as well as maternal and neonatal outcomes between the two groups. Women with gestational diabetes were excluded from our study. This study was approved by the Ethics Committee of the Institutional Review Board (CGH-IRB-2011-32) of Cheil General Hospital \& Women's Healthcare Center,
Seoul, Korea.

\section{Outcome measures}

Miscarriage was defined as the spontaneous ending of pregnancy before 20 weeks. Due to the increased risks associated with twin pregnancy, pregnancy and perinatal morbidity analyses were performed only in singleton pregnancies. Preterm delivery was defined as delivery before 37 weeks. For singleton infants, large for gestational age (LGA) was defined as a birth weight $\geq$ 9oth percentile and small for gestational age (SGA) as a birth weight $\leq$ 1oth percentile. Respiratory distress syndrome was defined as a breathing disorder that affected newborns and meconium aspiration syndrome was defined as a respiratory distress that developed shortly after birth, with radiographic evidence of aspiration pneumonia and the presence of meconium-stained amniotic fluid. Asphyxia was defined as the state of not being able to breathe. Jaundice was defined as a yellowish or greenish pigmentation of the skin and whites of the eyes due to high bilirubin levels [11]. Hypocalcemia was defined as the presence of low serum calcium levels in the blood (total serum $\mathrm{Ca}<7 \mathrm{mg} / \mathrm{dL}$ ). Hypoglycemia was defined as a reduction in blood sugar below normal levels (glucose $<35 \mathrm{mg} / \mathrm{dL}$ ). Cranial injury was defined as a history or physical signs of blunt trauma to the scalp, skull, or brain, and clavicle fracture was defined as a broken collarbone in a newborn baby that was just delivered. Macrosomia was defined as birth weight greater than 4,000 $\mathrm{g}$ regardless of gestational age. Major congenital malformations were classified according to the European Surveillance of Congenital Anomalies (EUROCAT) system [12]. Participants were classified as having type 2 diabetes or not according to their status before pregnancy. Maternal pre-pregnancy height and weight were obtained by a self-reported questionnaire.

\section{Statistical analysis}

Statistical analysis was performed with SPSS version 21.0 (IBM Co., Armonk, NY, USA). Analyses were performed using chi-square or Fisher exact tests for categorical variables, and independent $t$ tests for continuous variables. A $p<0.05$ was considered statistically significant. 


\section{RESULTS}

A total of 100 pairs of type 2 diabetic and nondiabetic pregnant women (200 women total) matched by age, pre-pregnancy weight, BMI, parity, and gestational age at delivery were compared. Maternal characteristics and glycated hemoglobin (HbAic) data are shown in Table 1. Details of the pregnancies and neonatal outcomes for the 200 singleton pregnancies are shown in Tables 2 and 3 .

\section{Demographics and HbAlc}

There were no significant differences between women with type 2 diabetes and nondiabetic controls in terms of maternal age $(33.8 \pm 4.5$ years vs. $33.7 \pm 3.7$ years, $p=$ 0.877 ), gestational age at delivery ( $38.7 \pm 1.6$ weeks vs. 38.7 \pm 2.4 weeks, $p=0.894)$, pregestational weight $(62.0 \pm 12.1$ kg vs. $62.8 \pm 10.2 \mathrm{~kg}, p=0.624$ ), parity (primipara: $52 \%$ vs. $50 \%, p=0.777$; multipara: $48 \%$ vs. $50 \%, p=0.777)$, and pregestational BMI $\left(24.1 \pm 4.0 \mathrm{~kg} / \mathrm{m}^{2}\right.$ vs. $24.2 \pm 3.8 \mathrm{~kg} / \mathrm{m}^{2}$, $p=0.803)$. Women with type 2 diabetes were more likely to have a family history of diabetes (78.0\% vs. $21.0 \%, p<$
0.001). In women with type 2 diabetes, their 1st trimester HbAlc was higher than their 2nd trimester HbAic (Table 1).

\section{Maternal outcomes}

We investigated maternal outcomes for the 200 pregnancies (Table 2). There were no miscarriages in any of the women with type 2 diabetes or the nondiabetic controls. Also, there were no terminations of pregnancy in either women with type 2 diabetes or nondiabetic controls.

Women with type 2 diabetes had a higher risk of primary C/S (35.0\% vs. 18.0\%, $p=0.006$ ). Pre-eclampsia was also more common in women with type 2 diabetes compared to nondiabetic controls $(10.0 \%$ vs. $2.0 \%, p=$ 0.017). There were also significant differences between women with type 2 diabetes and nondiabetic controls in terms of infections during pregnancy $(26.0 \%$ vs. $2.0 \%$, $p<0.001)$. There was no significant difference between women with type 2 diabetes and nondiabetic controls in terms of preterm delivery ( $9.0 \%$ vs. $10.0 \%, p=0.809$ ).

Table 1. Clinical features for nondiabetic control and type 2 diabetic pregnant women

\begin{tabular}{|c|c|c|c|}
\hline Variable & Nondiabetic controls & Type 2 diabetes & $p$ value \\
\hline Number & 100 & 100 & \\
\hline Maternal age, yr & $33.7 \pm 3.7$ & $33.8 \pm 4.5$ & 0.877 \\
\hline Gestational age at delivery, wk & $38.7 \pm 2.4$ & $38.7 \pm 1.6$ & 0.894 \\
\hline Pregestational weight, kg & $62.8 \pm 10.2$ & $62.0 \pm 12.1$ & 0.624 \\
\hline \multicolumn{4}{|l|}{ Parity } \\
\hline Primipara & $50(50.0)$ & $52(52.0)$ & 0.777 \\
\hline Multipara & $50(50.0)$ & $48(48.0)$ & 0.777 \\
\hline Pregestational BMI, kg/m² & $24.2 \pm 3.8$ & $24.1 \pm 4.0$ & \\
\hline Median (interquartile range) & $23.9(16.8-35.6)$ & $31(15-43)$ & 0.803 \\
\hline Age of diagnosis of DM, yr & - & $29.9 \pm 5.8$ & - \\
\hline Diabetes duration, yr & - & $3.7 \pm 4.2$ & - \\
\hline DM family history & $21(21.0)$ & $78(78.0)$ & $<0.001$ \\
\hline \multicolumn{4}{|l|}{$\mathrm{HbA1c}, \%^{\mathrm{a}}$} \\
\hline HbAic 1st trimester & - & $6.9 \pm 1.5$ & \\
\hline HbAıc and trimester & - & $5.7 \pm 1.0$ & \\
\hline HbAıc 3rd trimester & - & $6.1 \pm 0.8$ & \\
\hline
\end{tabular}

Values are presented as mean \pm SD or number (\%). The $p$ values represent overall differences across groups as determined by $t$ test for continuous variables and Pearson chi-square test for categorical variables.

BMI, body mass index; DM, diabetes mellitus; HbAic, glycated hemoglobin.

${ }^{\mathrm{a}} \mathrm{HbAic}$ was not investigated in the control group. 
Table 2. Maternal outcomes in nondiabetic controls and women with type 2 diabetes

\begin{tabular}{|c|c|c|c|}
\hline Variable & Nondiabetic controls & Type 2 diabetes & $p$ value \\
\hline Number & 100 & 100 & \\
\hline Miscarriage & o & o & - \\
\hline Termination of pregnancy & 0 & o & - \\
\hline \multicolumn{4}{|l|}{ Method of delivery } \\
\hline Vaginal delivery & $57(57.0)$ & $30(30.0)$ & $<0.001$ \\
\hline Induction & $1(1.0)$ & $1(1.0)$ & $1.000^{\mathrm{a}}$ \\
\hline Repeated C/S & $24(24.0)$ & $34(34.0)$ & 0.119 \\
\hline Primary C/S & $18(18.0)$ & $35(35 \cdot 0)$ & 0.006 \\
\hline Pre-eclampsia & $2(2.0)$ & $10(10.0)$ & $0.017^{\mathrm{a}}$ \\
\hline Infections during pregnancy & $2(2.0)$ & $26(26.0)$ & $<0.001^{\mathrm{a}}$ \\
\hline Preterm delivery < 37 weeks & $10(10.0)$ & $9(9.0)$ & 0.809 \\
\hline
\end{tabular}

Values are presented as number (\%).

$\mathrm{C} / \mathrm{S}$, caesarean section.

${ }^{a}$ The $p$ values represent overall differences between groups as determined by Fisher exact test.

\section{Neonatal outcomes}

Babies born to women with type 2 diabetes were more likely to be heavier $(3,370 \pm 552.0 \mathrm{~kg}$ vs. $3,196 \pm 543.3 \mathrm{~kg}$, $p=0.025)$. There were significant differences between women with type 2 diabetes and nondiabetic controls in terms of LGA babies (22.0\% vs. $9.0 \%, p=0.011)$, and macrosomic infants ( $15.0 \%$ vs. $5.0 \%, p=0.018)$. However, there were no significant differences between women with type 2 diabetes and nondiabetic controls in terms of SGA babies (7.0\% vs. 5.0\%, $p=0.552$ ).

There were no significant differences between women with type 2 diabetes and nondiabetic controls in Apgar score at 1 and 5 minutes $(8.0 \pm 1.0$ vs. $8.2 \pm 0.8, p=0.059$; $9.0 \pm 0.7$ vs. $9.0 \pm 0.7, p=0.487$ ). There were also no significant differences between women with type 2 diabetes and nondiabetic controls in terms of infants with respiratory distress $(40.0 \%$ vs. $28.0 \%, p=0.073)$ or jaundice (33.0\% vs. $21.0 \%, p=0.056$ ). There were two cases $(2.0 \%)$ of caudal regression syndrome among infants of women with type 2 diabetes and no congenital malformations in infants of the nondiabetic controls. There were no significant differences between women with type 2 diabetes and nondiabetic controls in terms of neonatal intensive care unit admission (14.0\% vs. 13.0\%, $p=0.836$ ) (Table 3 ).

\section{DISCUSSION}

We found that maternal and neonatal outcomes in women with type 2 diabetes were worse than those of women without diabetes, especially in terms of the need for a primary $\mathrm{C} / \mathrm{S}$, pre-eclampsia, infections during pregnancy, neonatal weight, LGA, and macrosomia.

A previous study showed that women with type 2 diabetes are at greater risk for developing preeclampsia, preterm labor, intrauterine growth restriction, in utero fetal demise, fetal congenital anomalies, macrosomia, shoulder dystocia, and the need for a cesarean delivery [8-10]. We observed a significant difference in the rate of primary cesarean section between women with type 2 diabetes and women without diabetes. These data are in accordance with a previous study that showed that women with pre-existing diabetes mellitus had a significantly greater rate of primary cesarean section compared to nondiabetic controls [10,13,14]. Knight et al. [15] also reported that women with type 2 diabetes were more likely to deliver via cesarean section than women without diabetes.

Consistent with our results, Knight et al. [15] showed that women with type 2 diabetes were more likely to have LGA and macrosomic infants than women without diabetes. Macrosomic infants are more often delivered by cesarean section because of failed progress in labor or in order to prevent shoulder dystocia $[13,14,16]$. In an- 
Table 3. Neonatal outcomes in nondiabetic controls and women with type 2 diabetes

\begin{tabular}{|c|c|c|c|}
\hline Variable & Nondiabetic controls & Type 2 diabetes & $p$ value \\
\hline Number & 100 & 100 & \\
\hline Neonatal weight, g & $3,196 \pm 543 \cdot 3$ & $3,370 \pm 552.0$ & 0.025 \\
\hline LGA $\geq$ 9oth percentile & $9(9.0)$ & $22(22.0)$ & 0.011 \\
\hline SGA $\leq$ 1oth percentile & $5(5.0)$ & $7(7.0)$ & $0.55^{2}$ \\
\hline Macrosomia & $5(5 \cdot 0)$ & $15(15 \cdot 0)$ & 0.018 \\
\hline Apgar score at 1 minute & $8.2 \pm 0.8$ & $8.0 \pm 1.0$ & 0.059 \\
\hline Median (interquartile range) & $8(5-10)$ & $8(2-10)$ & \\
\hline Apgar score at 5 minutes & $9.0 \pm 0.7$ & $9.0 \pm 0.7$ & 0.487 \\
\hline Median (interquartile range) & $9(7-10)$ & $9(6-10)$ & \\
\hline \multicolumn{4}{|l|}{ Neonatal complications } \\
\hline RDS & $28(28.0)$ & $40(40.0)$ & 0.073 \\
\hline MAS & $5(5.0)$ & $1(1.0)$ & $0.212^{\mathrm{a}}$ \\
\hline Asphyxia & $1(1.0)$ & $1(1.0)$ & $1.000^{\mathrm{a}}$ \\
\hline Hyperbilirubinemia, jaundice & $21(21.0)$ & $33(33 \cdot 0)$ & 0.056 \\
\hline Hypocalcemia & $\mathrm{O}$ & $3(3 \cdot 0)$ & $0.246^{\mathrm{a}}$ \\
\hline Hypoglycemia & 0 & $4(4 \cdot 0)$ & $0.121^{\mathrm{a}}$ \\
\hline Cranial injury & $1(1.0)$ & $3(3.0)$ & $0.621^{a}$ \\
\hline Clavicle fracture & $1(1.0)$ & $2(2.0)$ & $1.000^{\mathrm{a}}$ \\
\hline Malformation (caudal regression) & 0 & $2(2.0)$ & $0.497^{\mathrm{a}}$ \\
\hline NICU admission & $13(13.0)$ & $14(14.0)$ & 0.836 \\
\hline
\end{tabular}

Values are presented as mean \pm SD or number (\%). The $p$ values represent overall differences across groups as determined by $t$ test for continuous variables and Pearson chi-square test for categorical variables.

LGA, large for gestational age; SGA, small for gestational age; RDS, respiratory distress syndrome; MAS, meconium aspiration syndrome; NICU, neonatal intensive care unit.

${ }^{\mathrm{a}}$ Fisher exact test.

other study, macrosomic infants experienced more frequent birth trauma and shoulder dystocia, higher death rates, and lower Apgar scores [17]. This finding suggests that more intensive prenatal care is needed to improve glycemic control and perinatal outcomes in patients with type 2 diabetes. One possible method is continuous glucose monitoring (CGM) during pregnancy, as randomized clinical trials using CGM during pregnancy demonstrated improved glycemic control and reduced frequency of LGA infants in women with type 1 and type 2 diabetes [18].

Preliminary studies indicate that diabetes mellitus delays maturation of the lecithin/sphingomyelin ratio (L/S ratio) [19]. In normal pregnancies, the L/S ratio correlates with gestational age. In the pregnancies of diabetic women, this correlation is low, and fetal lung maturation may be delayed [20]. The association between glycemic con- trol and congenital malformations has been consistently documented [21,22]. Therefore, maternal glucose is considered to be an important factor and diabetic patients should be encouraged to optimize glucose control. We found no significant difference in the rates of congenital malformation between the two groups; however, this study did not have a large enough sample size to show such differences between the two groups.

In summary, we found that Korean women with type 2 diabetes had worse maternal and neonatal outcomes compared to women without diabetes, especially in terms of primary $\mathrm{C} / \mathrm{S}$, pre-eclampsia, infections in pregnancy, neonatal weight, LGA, and macrosomia. It may be difficult to generalize these findings since the data were collected at a single center. Future multicenter studies with larger samples of type 2 diabetic and nondiabetic women are necessary to validate these results. 
The results of this study show the need for hospital and regional participation to recruit a larger cohort necessary for precise investigation of pregnancy outcomes in Korean women. This will improve our ability to counsel and manage these patients, properly direct them to helpful resources, and identify chances for improving their care in order to optimize maternal and neonatal outcomes.

\section{KEY MESSAGE}

1. Maternal and neonatal outcomes in Korean women with type 2 diabetes were worse than those without diabetes, especially in terms of primary caesarean section, pre-eclampsia, infections in pregnancy, large for gestational age, and macrosomia.

2. Further studies are needed to improve pregnancy outcomes in women with type 2 diabetes, such as prepregnancy care and more meticulous glucose control.

\section{Conflict of interest}

No potential conflict of interest relevant to this article was reported.

\section{REFERENCES}

1. Wild S, Roglic G, Green A, Sicree R, King H. Global prevalence of diabetes: estimates for the year 2000 and projections for 2030. Diabetes Care 2004;27:1047-1053.

2. Dunstan DW, Zimmet PZ, Welborn TA, et al. The rising prevalence of diabetes and impaired glucose tolerance: the Australian Diabetes, Obesity and Lifestyle Study. Diabetes Care 2002;25:829-834.

3. Confidential Enquiry into Maternal and Child Health (CEMACH). Pregnancy in Women with Type 1 and Type 2 Diabetes 2002-2003: England, Wales and Northern Ireland. London: CEMACH, 2005 .

4. Lawrence JM, Contreras R, Chen W, Sacks DA. Trends in the prevalence of preexisting diabetes and gestational diabetes mellitus among a racially/ethnically diverse population of pregnant women, 1999-2005. Diabetes Care 2008;31:899-904.
5. Verheijen EC, Critchley JA, Whitelaw DC, Tuffnell DJ. Outcomes of pregnancies in women with pre-existing type 1 or type 2 diabetes, in an ethnically mixed population. BJOG 2005;112:1500-1503.

6. Brydon P, Smith T, Proffitt M, Gee H, Holder R, Dunne F. Pregnancy outcome in women with type 2 diabetes mellitus needs to be addressed. Int J Clin Pract 2000;54:418419.

7. Stamler EF, Cruz ML, Mimouni F, et al. High infectious morbidity in pregnant women with insulin-dependent diabetes: an understated complication. Am J Obstet Gynecol 1990;163(4 Pt 1):1217-1221.

8. Macintosh MC, Fleming KM, Bailey JA, et al. Perinatal mortality and congenital anomalies in babies of women with type 1 or type 2 diabetes in England, Wales, and Northern Ireland: population based study. BMJ 2006;333:177.

9. Kitzmiller JL, Buchanan TA, Kjos S, Combs CA, Ratner RE. Pre-conception care of diabetes, congenital malformations, and spontaneous abortions. Diabetes Care 1996;19:514-541.

10. Remsberg KE, McKeown RE, McFarland KF, Irwin LS. Diabetes in pregnancy and cesarean delivery. Diabetes Care 1999;22:1561-1567.

11. Buttaro TM, Trybulski J, Polgar-Bailey P, Sandberg-Cook J. Primary Care: A Collaborative Practice. 4 th ed. St. Louis: Elsevier/Mosby, 2013.

12. Lechat MF, Dolk H. Registries of congenital anomalies: EUROCAT. Environ Health Perspect 1993;101 Suppl 2:153157.

13. Wahabi HA, Esmaeil SA, Fayed A, Al-Shaikh G, Alzeidan RA. Pre-existing diabetes mellitus and adverse pregnancy outcomes. BMC Res Notes 2012;5:496.

14. MacDorman MF, Menacker F, Declercq E. Cesarean birth in the United States: epidemiology, trends, and outcomes. Clin Perinatol 2008;35:293-307.

15. Knight KM, Pressman EK, Hackney DN, Thornburg LL. Perinatal outcomes in type 2 diabetic patients compared with non-diabetic patients matched by body mass index. J Matern Fetal Neonatal Med 2012;25:611-615.

16. Modanlou HD, Komatsu G, Dorchester W, Freeman RK, Bosu SK. Large-for-gestational-age neonates: anthropometric reasons for shoulder dystocia. Obstet Gynecol 1982;60:417-423.

17. Spellacy WN, Miller S, Winegar A, Peterson PQ. Macrosomia: maternal characteristics and infant complications. 
Obstet Gynecol 1985;66:158-161.

18. Murphy HR, Rayman G, Lewis K, et al. Effectiveness of continuous glucose monitoring in pregnant women with diabetes: randomised clinical trial. BMJ 2008;337:a1680.

19. Gluck L, Kulovich MV. Lecithin-sphingomyelin ratios in amniotic fluid in normal and abnormal pregnancy. Am J Obstet Gynecol 1973;115:539-546.

20. Gluck L, Kulovich MV, Eidelman AI, Cordero L, Khazin AF. Biochemical development of surface activity in mammalian lung. IV. Pulmonary lecithin synthesis in the human fetus and newborn and etiology of the respiratory distress syndrome. Pediatr Res 1972;6:81-99.

21. Inkster ME, Fahey TP, Donnan PT, Leese GP, Mires GJ, Murphy DJ. Poor glycated haemoglobin control and adverse pregnancy outcomes in type 1 and type 2 diabetes mellitus: systematic review of observational studies. BMC Pregnancy Childbirth 2006;6:30.

22. Klingensmith GJ, Pyle L, Nadeau KJ, et al. Pregnancy outcomes in youth with type 2 diabetes: the TODAY study experience. Diabetes Care 2016;39:122-129. 